\title{
Optimisation of absorber parameters in the case of stochastic vibrations in a bridge with a deck platform for servicing pipelines
}

https://doi.org/10.2478/sgem-2021-0030

received August 17, 2021; accepted October 26, 2021.

\begin{abstract}
The paper focuses on the problem of optimising the cooperation between a dynamic vibration absorber (DVA) and a structure. The authors analyse a road beam bridge equipped with a working platform (deck) used to service pipelines installed on the structure. The paper studies the problem of choosing the optimal parameters for damping absorbers that reduce the random vibration of a beam subjected to a random sequence of moving forces with a constant velocity. The stochastic properties of the load are modelled by means of a filtering Poisson process. A single-degree-of-freedom (SDOF) absorber model with a multi-degree-of-freedom (MDOF) primary structure model are is considered.
\end{abstract}

Keywords: urban network infrastructure; dynamic vibration absorber (DVA); vibration effect; dynamic analysis; Poisson process; random vibration.

\section{Introduction}

The problem of reducing the level of vibrations in various constructions has been considered for many years. This is especially the case for classic bridge structures when an additional platform on which installations, for example, pipes with hazardous gas, are installed.

Numerous ways and means of preventing unacceptable vibrations are known, with one of these methods being use of various vibration absorbers. Their application has a special role in civil engineering due

\footnotetext{
*Corresponding author: Monika Podwórna, Faculty of Civil Engineering, Wroclaw University of Science and Technology, Wyb. Wyspiańskiego 27, 50-370 Wroclaw, Poland, E-mail: monika.podworna@pwr.edu.pl Jacek Grosel, Faculty of Civil Engineering, Wroclaw University of Science and Technology, Wyb. Wyspiańskiego 27, 50-370 Wroclaw, Poland
}

to the fact that they can be used during a construction's design, as well as later on to modify the structure. Using absorbers is an interesting option for reducing the vibrations of various types of structures, especially in tall buildings [3] or in bridges, for example, the Millennium Bridge [1] in London or bridges traversed by high-speed trains [2]. Moreover, absorbers can be used to prevent large vibration amplitudes that occasionally appear, that is, in geotechnical works [4].

The topic of dynamic vibration absorbers (DVAs) or tuned mass dampers (TMD) is well established in literature. The action of the absorber is accepted as a deterministic [5-9] or random process [10-20]. A large number of studies have been conducted to optimise the design parameters, and hence maximise the performance of vibration absorbers. Although the design concept of a TMD was developed decades ago, its simplicity and effectiveness have made it one of the most popular passive vibration methods to suppress structural vibration [21].

In the earlier years of the 20th century, Frahm [22] introduced the use of a linear spring mass attachment to suppress the oscillations of harmonically excited primary structural systems in engineering applications. This early DVA was able to reduce the oscillations of primary structures with a single degree of freedom, but could only reduce vibration transmission in a specific narrow frequency band. A spring-supported mass invented by Frahm is a dynamic absorber without damping, known as a Frahm damper. Ormondroyd and Den Hartog [23] increased the effectiveness of the absorber to dissipate the energy of the primary structure to harmonic excitations by appending a viscous damper parallel to the linear spring. Later, a semi-empirical design procedure was established by Den Hartog [5]. The DVA proposed by Den Hartog is now known as the Voigt-type DVA dynamic vibration absorber, where a spring element and a viscous element are connected. This is considered as the standard model of the DVA. The main objective in the design of the standard type DVA is to enable the absorber to have optimum 




Figure 1: The model of the system.

parameters. Due to the fact that the mass ratio of the DVA to the primary structure is usually a few percent, the principal parameters of the DVA are its tuning ratio (i.e. the ratio of the DVA's frequency to the natural frequency of the primary structure) and damping ratio [24].

Ormondroyd and Den Hartog [23] developed analytical solutions for the optimal design of the classic DVA using the fixed-points technique. Den Hartog [5] provided an algorithm for selecting the optimal absorber parameters, so that the maximum amplitude of vibration is minimised over frequencies of deterministic sinusoidal excitations, which is essentially an $\mathrm{H}_{1}$ optimisation. Asami and Nishihara used $\mathrm{H}_{\infty}$ and $\mathrm{H}_{2}$ optimisation methods based on the perturbation technique and Vieta's theory to derive analytical solutions for the optimal design of the classic DVA [25-26]. Sims [27] introduced a new analytical solution based on the criterion of minimising either the positive real part or the negative real part of the frequency response function. Shen et al. [28] proposed a new strategy for obtaining the optimum negative stiffness ratio and to also make the system remain stable.

Most works assume that the load process is deterministic and that it changes harmonically. The problem of the optimisation of absorbers connected with a bridge beam loaded by a moving force was considered in papers [29-30]. The vibrations of some structures are excited by a load of random nature. There are relatively few studies on absorbers in which the load is a stationary stochastic process [31-34]. In the mentioned works, stochastic vibrations were analysed in the field of correlation theory and spectral density analysis.

The earliest research works on the optimal design of a DVA were presented for a single degree of freedom (SDOF) of a primary structure $[5,22,23]$. In the vast majority of papers, the structure-absorber system is still treated as a system with two degrees of freedom (2DOF). The optimal design of an absorber is not a new topic, and the optimal methodologies and parameters of the optimal DVA or TMD system for SDOF main structures subjected to different loading conditions have been verified by several researchers since the 1960s [21].

The problem of choosing the optimal parameters for damping absorbers that reduce the random vibrations of a beam subjected to a sequence of moving forces with a constant velocity is studied in this paper. Every force is regarded as a random variable. Moreover, the inter-arrival times of moving forces are regarded as random variables. The stochastic properties of the load are modelled by means of a filtered Poisson process. The problem was solved with the idea of a dynamic influence function [35]. Several optimisation criteria, based on the expected values and variance of the beam response determined in the study, were considered.

\section{Formulation of the problem and general solutions}

Let us consider the damping vibrations of a simply supported Euler-Bernoulli beam of finite length $L$ with an absorber fitted at point $x_{a}$ subjected to a sequence of moving forces with a constant velocity $v$ (see Figure 1).

The vibrations of the beam are described by the equation [36]

$$
\begin{gathered}
E I \frac{\partial^{4} w(x, t)}{\partial x^{4}}+c \frac{\partial w(x, t)}{\partial t}+m \frac{\partial^{2} w(x, t)}{\partial t^{2}}+r(t) \delta\left(x-x_{a}\right)= \\
\sum_{k=1}^{N(t)} A_{k} \delta\left[x-v\left(t-t_{k}\right)\right]
\end{gathered}
$$

where $E I$ denotes the flexural stiffness of the beam, $m$ denotes the mass per unit length of the beam, $c$ is the 
damping coefficient, $\delta($.$) denotes the Dirac delta function$ and $r(t)$ is the force response of the absorber acting on the beam. The reaction of absorber on beam can be derived from equilibrium of the absorber.

The amplitudes $A_{k}$ are assumed to be random variables, which are both mutually independent and independent of the random instants $t_{k}$. It is assumed that the expected values $E\left[A_{k}\right]=E[A]=$ const are known. Random times $t_{k}$ constitute a Poisson process $N(t)$ with a parameter $\lambda$.

For a finite, simply supported beam, the boundary conditions have the following forms:

$$
\begin{gathered}
w(0, t)=w(L, t)=0, \\
\left.\frac{\partial^{2} w(x, t)}{\partial x^{2}}\right|_{x=0}=\left.\frac{\partial^{2} w(x, t)}{\partial x^{2}}\right|_{x=L}=0 .
\end{gathered}
$$

Let us introduce two dynamic influence functions $-H_{1}(x, t)$ and $H_{2}(x, t-L / v)$. The function $H_{1}(x, t)$ is the response of the beam at time $t(0 \leq t \leq L / v)$ to a moving force equal to unity $\left(A_{k}=1\right)$, and the function $H_{2}(x, t-L / v)$ is the response of the system without excitation, but with non-zero initial conditions (the force has already left the beam). These influence functions satisfy the following differential equations:

$$
\begin{gathered}
E I \frac{\partial^{4} H_{1}(x, t)}{\partial x^{4}}+c \frac{\partial H_{1}(x, t)}{\partial t}+m \frac{\partial^{2} H_{1}(x, t)}{\partial t^{2}}+ \\
r(t) \delta\left(x-x_{a}\right)=\delta(x-v t), \\
E I \frac{\partial^{4} H_{2}\left(x, t-\frac{L}{v}\right)}{\partial x^{4}}+c \frac{\partial H_{2}\left(x, t-\frac{L}{v}\right)}{\partial t}+m \frac{\partial^{2} H_{2}\left(x, t-\frac{L}{v}\right)}{\partial t^{2}}+ \\
+r\left(t-\frac{L}{v}\right) \delta\left(x-x_{a}\right)=0,
\end{gathered}
$$

and appropriate boundary conditions and initial conditions

$$
\begin{gathered}
H_{1}(x, 0)=\left.\frac{\partial H_{1}(x, t)}{\partial t}\right|_{t=0}=0, \\
H_{2}(x, 0)=H_{1}\left(x, \frac{L}{v}\right),\left.\frac{\partial H_{2}(x, t)}{\partial t}\right|_{t=0}=\left.\frac{\partial H_{1}(x, t)}{\partial t}\right|_{t=\frac{L}{v}} .
\end{gathered}
$$

The deflection of the beam $w_{I}(x, t)$ is counted at arbitrary time $t$, and none of the force locations are known. It can be written in the Stieltjes integral:

$$
w_{I}(x, t)=\int_{t-\frac{L}{v}}^{t} A(\tau) H_{1}(x, t-\tau) d N(\tau)+
$$

$$
+\int_{t_{b}}^{t-\frac{L}{v}} A(\tau) H_{2}\left(x, t-\tau-\frac{L}{v}\right) d N(\tau)
$$

The expected value of the random function $w_{I}(x, t)$ amounts to [37]

$$
\begin{gathered}
E\left[w_{I}(x, t)\right]=E[A] \lambda \int_{t-\frac{L}{v}}^{t} H_{1}(x, t-\tau) d \tau+ \\
E[A] \lambda \int_{t_{b}}^{t-\frac{L}{v}} H_{2}\left(x, t-\tau-\frac{L}{v}\right) d \tau
\end{gathered}
$$

whereas the variance amounts to

$$
\begin{gathered}
\sigma_{w I}^{2}(x, t)=E\left[A^{2}\right] \lambda \int_{t-\frac{L}{v}}^{t} H_{1}^{2}(x, t-\tau) d \tau+ \\
E\left[A^{2}\right] \lambda \int_{t_{b}}^{t-\frac{L}{v}} H_{2}^{2}\left(x, t-\tau-\frac{L}{v}\right) d \tau .
\end{gathered}
$$

The symbol $E[$.] denotes the expected value of the quantity in the brackets, $v_{A}$ is the coefficient of variation and $E\left[A^{2}\right]=E^{2}[A]\left(1+v_{A}^{2}\right)$. The parameter $\lambda>0$ is the intensity of the Poisson process and is equal to the average number of points per unit of time. The above general solution will be used to optimise the absorber parameters. For transient vibration, we assume $t_{b}=0$ and for steady state, $t_{b}=-\infty$. In the last case, equations (9) and (10) have the forms

$$
\begin{gathered}
E\left[w_{I}(x, \infty)\right]=E[A] \lambda \int_{0}^{\frac{L}{v}} H_{1}(x, \tau) d \tau+ \\
+E[A] \lambda \int_{\frac{L}{v}}^{\infty} H_{2}\left(x, t-\tau-\frac{L}{v}\right) d \tau= \\
=E[A] \lambda E[w]
\end{gathered}
$$

$$
\begin{gathered}
\sigma_{w I}^{2}(x, \infty)=E\left[A^{2}\right] \lambda \int_{0}^{\frac{L}{v}} H_{1}^{2}(x, \tau) d \tau+ \\
+E\left[A^{2}\right] \lambda \int_{\frac{L}{v}}^{\infty} H_{2}^{2}\left(x, t-\tau-\frac{L}{v}\right) d \tau= \\
=E\left[A^{2}\right] \lambda \sigma^{2} .
\end{gathered}
$$

\section{Determination of the dynamic influence function}

In the case of a simply supported beam, one can look for dynamic influence functions in the form of sine series 


$$
\begin{gathered}
H_{1}(x, t)=\sum_{n=1}^{\infty} y_{1 n}(t) \sin \frac{n \pi x}{L}, \\
H_{2}\left(x, t-\frac{L}{v}\right)=\sum_{n=1}^{\infty} y_{2 n}\left(t-\frac{L}{v}\right) \sin \frac{n \pi x}{L} .
\end{gathered}
$$

By substituting expressions (13) and (14) into equations (4) and (5), and using the orthogonality method (multiplying by $\sin \frac{n \pi x}{L}$, integrating over $0 \leq x \leq L$ and taking into consideration $j$ first eigenforms), one can obtain the following sets of ordinary equations:

$$
\begin{gathered}
\frac{d^{2} y_{1 n}(t)}{d t^{2}}+2 \alpha \frac{d y_{1 n}(t)}{d t}+\omega_{n}^{2} y_{1 n}(t)+ \\
+r(t) \sin \frac{n \pi x_{a}}{L}=\frac{2}{m L} \sin \frac{n \pi v t}{L}, \\
\frac{d^{2} y_{2 n}(t)}{d t^{2}}+2 \alpha \frac{d y_{2 n}(t)}{d t}+\omega_{n}^{2} y_{2 n}(t)+r(t) \sin \frac{n \pi x_{a}}{L}=0,
\end{gathered}
$$

where $=1,2, \ldots, j, 2 \alpha=\frac{c}{m}, \omega_{n}^{2}=\left(\frac{n \pi}{L}\right)^{4} \frac{E I}{m}$. The parameter $j$ in the tests varied from 1 to 20 , while for further calculations, it was found that a sufficient number was $j$ $=5$. The initial conditions for the functions $y_{1 n}(t)$ and $y_{2 n}(t)$ result directly from conditions (6) and (7).

\section{Absorber equations}

The equations of motion and the $r(t)$ reaction of their impact on the beam will be derived. The vibrations of the SDOF absorber attached to the beam at point $x_{a}$ are described by the following equation:

$$
\begin{gathered}
M_{a} \frac{d^{2} q_{a}(t)}{d t^{2}}+c_{a}\left[\frac{d q_{a}(t)}{d t}-\frac{d H_{i}\left(x_{a}, t\right)}{d t}\right]+ \\
+k_{a}\left[q_{a}(t)-H_{i}\left(x_{a}, t\right)\right]=0,
\end{gathered}
$$

which, after taking into account (13) or (14) and the $j$ first eigenforms, has the form

$$
\begin{gathered}
\frac{d^{2} q_{a}(t)}{d t^{2}}+2 \alpha_{a}\left[\frac{d q_{a}(t)}{d t}-\sum_{n=1}^{j} \frac{d y_{i n}(t)}{d t} \sin \frac{n \pi x_{a}}{L}\right]+ \\
+\omega_{a}^{2}\left[q_{a}(t)-\sum_{n=1}^{j} y_{i n}(t) \sin \frac{n \pi x_{a}}{L}\right]=0
\end{gathered},
$$

where $i=1,2$ and $2 \alpha_{a}=\frac{c_{a}}{M_{a}}, \omega_{a}^{2}=\frac{k_{a}}{M_{a}}$.
The reaction $r(t)$ between the beam and the absorber is given by the following equation:

$$
r(t)=M_{a} \frac{d^{2} q_{a}(t)}{d t^{2}}
$$

It is assumed that the reaction $r(t)$ acts upwards on the beam. After substituting dependence (19) to equations (15) and (16), two separate systems of ordinary differential equations, together with equation (18), are obtained, for which solutions are achieved by numerical integration using Wolfram Mathematica [38]. As a standard, this software uses the Runge-Kutta method, automatically selecting the integration step; in the calculations, the maximum integration step was limited to one-thousandth of the passing time.

\section{Optimisation criteria and reliability assessments}

The issue of optimising absorber parameters has been considered in many studies. The problem cannot always be solved in an analytical way, especially in the case of stochastic loads. Moreover, it is possible to adopt various optimisation criteria. The solution of the optimisation problem depends both on the speed of the moving force and the parameters of the beam.

Let us assume the following optimisation criteria:

$$
\begin{gathered}
\operatorname{Min} E\left[w_{I}(x, \infty)\right]=\operatorname{Min} E[w], \\
\operatorname{Min} \sigma_{w I}^{2}(x, \infty)=\operatorname{Min} \sigma^{2} .
\end{gathered}
$$

Let $h$ denote the maximum displacement of the beam in section $x$ (e.g. $x=0.5 L$ ), which is caused by the passage of a unit force $(A=1)$ :

$$
h=H_{1}\left(x, t_{i}\right)=\operatorname{Max} H_{1}(x, t) .
$$

The knowledge of the quantity $h$ allows the problem of parameter optimisation in terms of assessing the beam's reliability to be formulated. Let us assume that $F_{A}(x)$ denotes the log-normal distribution function of the random amplitude $A_{i}$, of which the expected value and variance are known. A force of $A_{i}$ amplitude causes 
maximum displacement of the beam equal to $A_{i} h$. Let function $F(x, t)_{\max }$ determine the distribution of beam response maxima in a given period $(0, t)$ It is assumed that the time of a single passage is small enough, when compared to the time of exploiting the structure, that it can be omitted, and therefore, a single passage can be treated as an impulse. This function is given by the equation [39]:

$$
\begin{gathered}
F_{\max }(x, t)=\sum_{i=1}^{\infty} P\{N(t)= \\
=k\} \prod_{i=1}^{j}\left\{P\left(A_{i} \leq \frac{x}{h}\right)\right\}=\sum_{j=1}^{\infty} p_{n}(t) F_{A}^{j}\left(\frac{x}{h}\right),
\end{gathered}
$$

where $p_{n}(t)$ is the probability of $j$ pulses in time $(0, t)$.

Considering that the $N(t)$ process is a Poisson process with parameter $\lambda>0$, the above equation can be presented in the form [39]:

$$
F_{\max }(x, t)=\exp \left\{-\lambda t\left[1-F_{A}\left(\frac{x}{h}\right)\right]\right\}
$$

The above solutions can be used to optimise the parameters of the absorber with respect to reliability. As the first criterion, we can assume a minimum probability of exceeding the permissible level $\Delta$ by a single pulse. This probability $p_{f}$ is given by the equation [39]:

$$
p_{f}=1-p_{r}=1-\Phi\left[\frac{\ln \left(\frac{\Delta}{h}\right)-E[\ln A]}{\sigma_{\ln A}^{2}}\right]
$$

where $\Phi($.$) is the standard normal distribution.$

The next criterion assumes the probability that the maximum vibrations in the period $(0, T)$ will not exceed $\Delta$ level. This probability is equal to [39]:

$$
p_{r}(T)=1-p_{f}(T)=\exp \left\{-\lambda T\left[\left[1-F_{A}\left(\frac{\Delta}{h}\right)\right]\right\},\right.
$$

where

$$
F_{A}\left(\frac{\Delta}{h}\right)=\Phi\left[\frac{\ln \left(\frac{\Delta}{h}\right)-E[\ln A]}{\sigma_{\ln A}^{2}}\right]
$$

and $\sigma_{\ln A}^{2}=\ln \left(1+v_{A}^{2}\right), E[\ln A]=m_{\ln A}=\ln (E[A])-\frac{1}{2} \sigma_{A}^{2}$.



Figure 2: Deflection of the structure. An absorber with parameters $\mu=0.05, \kappa=1$.

\section{Numerical analysis}

The model of the structure refers to a road bridge with a suspended platform equipped with municipal technical infrastructure. It is a reinforced concrete beam bridge with a span of $L=30 \mathrm{~m}$, in which the first eigenfrequency $\omega_{s}=4$ $\mathrm{rad} / \mathrm{s}$. After analysing a range of 3-20 forms, it was found that 5 eigenforms can be adopted for further calculations. Different positions of the absorbers, $x_{a} \in(0, L)$, were considered. Various parameters of the absorber were analysed $\left(\mu=M_{a} / m L, \kappa=\omega_{a} / \omega_{s}\right)$. Since $E[A], E\left[A^{2}\right]$ and $\lambda$ are constant for the homogeneous Poisson process, the figures below show the deflections, expected values and variances of displacements in the middle of the span of the bridge beam without the value of these parameters.

Figures 2 and 3 show that the deflection of the structure is in three places $\left(x_{a}=L / 4, L / 2,3 / 4 L\right)$. Figure 2 shows deflections of the structure with the absorber $\left(\mu=M_{a} I\right.$ $\left.m L=0.05, \kappa=\omega_{a} / \omega_{s}=1\right)$. It was assumed that the vehicle traffic moves at a critical speed, which was adopted according to the well-known formula $\left(v_{c r}=\pi \sqrt{E I / m L^{2}}\right)$.

As shown in the figure above, the beam deflection in one-fourth of its length on the left and right sides is almost identical after the travel time has elapsed. Slight changes are visible at the beginning of the observation. A similar situation can be seen for the structure without an absorber (see Figure 3). It is obvious that deflection in the middle is greater than at one-fourth of the beam's length.

Various tuned absorbers were analysed. The influence of $\kappa=\omega_{a} / \omega_{s}$ parameter is definitely greater than that of mass $\mu=M_{a} / m L$. Figure 4 shows three deflections in the middle of the beam: the blue line is for a structure without an absorber, the orange line is for an absorber with parameters $\mu=0.05, \kappa=1$ and the green line is for $\mu=0.05$, $\kappa=2$.

Figure 5 shows three deflections in the middle of the beam: the blue line is for a structure without an absorber, 


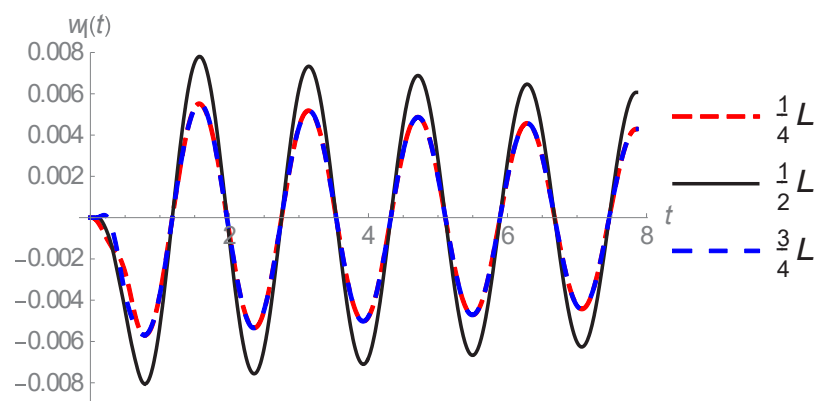

Figure 3: Deflection of the structure without an absorber.



Figure 4: Deflection of the structure in the midspan for two absorbers with different $\kappa$.



Figure 5: Deflection of the structure in the midspan for two absorbers with different $\mu$.

the orange line is for an absorber with parameters $\mu=0.05$, $\kappa=1$ and the green line is for $\mu=0.025, \kappa=1$.

The graphs below show the analysis of the absorber with the parameters $\mu=0.05, \kappa=1$. When comparing Figures $3-5$, it can be seen that the absorber is well-tuned, which means that it reduces the vibrations of the beam. The influence of the velocity of mobile vehicles was also analysed (see Figures 6 and 7).

A wide range of analysis of the influence of the absorber mass was adopted for $\mu \in\langle 0,0.3\rangle$. In practice, up to $10 \%$ is usually used $\left(\mu_{\max }=0.1\right)$. As shown in Figure $8 \mathrm{a}$, the expected value of the displacement in the middle of the span of the bridge beam, along with the equipment and technical infrastructure, does not depend on the absorber's mass. Similarly, a wide range of analysis of the influence of the absorber frequency was adopted,

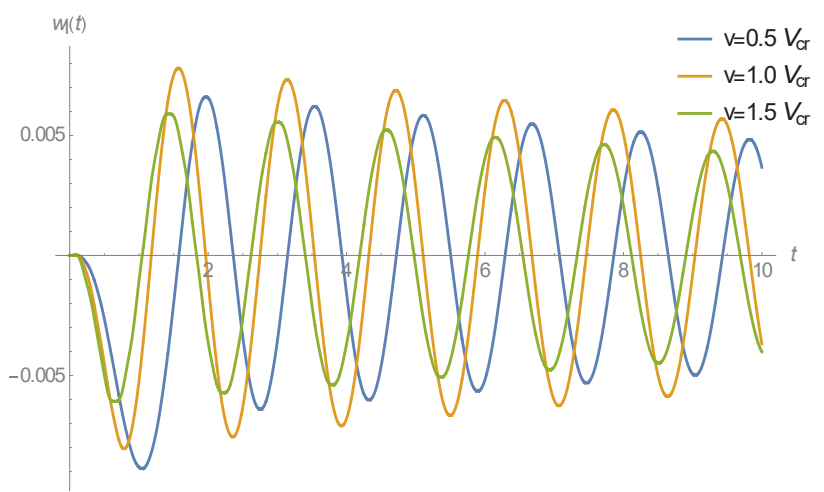

Figure 6: Deflection of the structure without an absorber in the middle of the beam for three velocities.

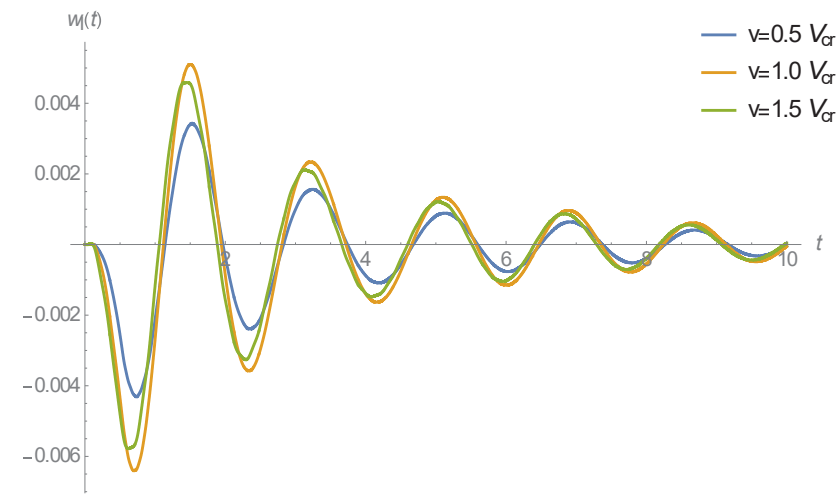

Figure 7: Deflection of the structure with the absorber $\mu=0.05, k=1$ in the middle of the beam for three velocities.

$\kappa \in\langle 0.5,2.5\rangle$. In practice, an absorber with a frequency similar to the structure's frequency $(\kappa \approx 1)$ is most often used. As shown in Figure $8 \mathrm{~b}$, the expected value of the displacement in the midspan does not depend on the absorber's frequency. However, the impact of the velocity of cars is very large. The analysed range for the selected structure is $68-336 \mathrm{~km} / \mathrm{h}$. A higher speed corresponds to a smaller deflection (the greater the influence of the absorber).

As shown in Figure 9a and b, the variance of these displacements also depends on velocity, but in a different way than is expected. The biggest variance of deflection is when the velocity of the passage is within the range 0.6-0.8 $v_{c r}$ with regards to the analysis of both the $\mu$ and $\kappa$ parameters. However, there is a slight influence of the mass ratio (see Figure 9a). Good results can be seen for $\mu \in\langle 5 \%, 15 \%\rangle$. When $\mu<5 \%$ and $\mu>20 \%$ for $v=(0.6-0.8) v_{c r}$, the variance increases. The influence of the frequency ratio can also be seen (see Figure 9b). The smaller the $\kappa$, the smaller the deflection is in the range of critical velocity. 

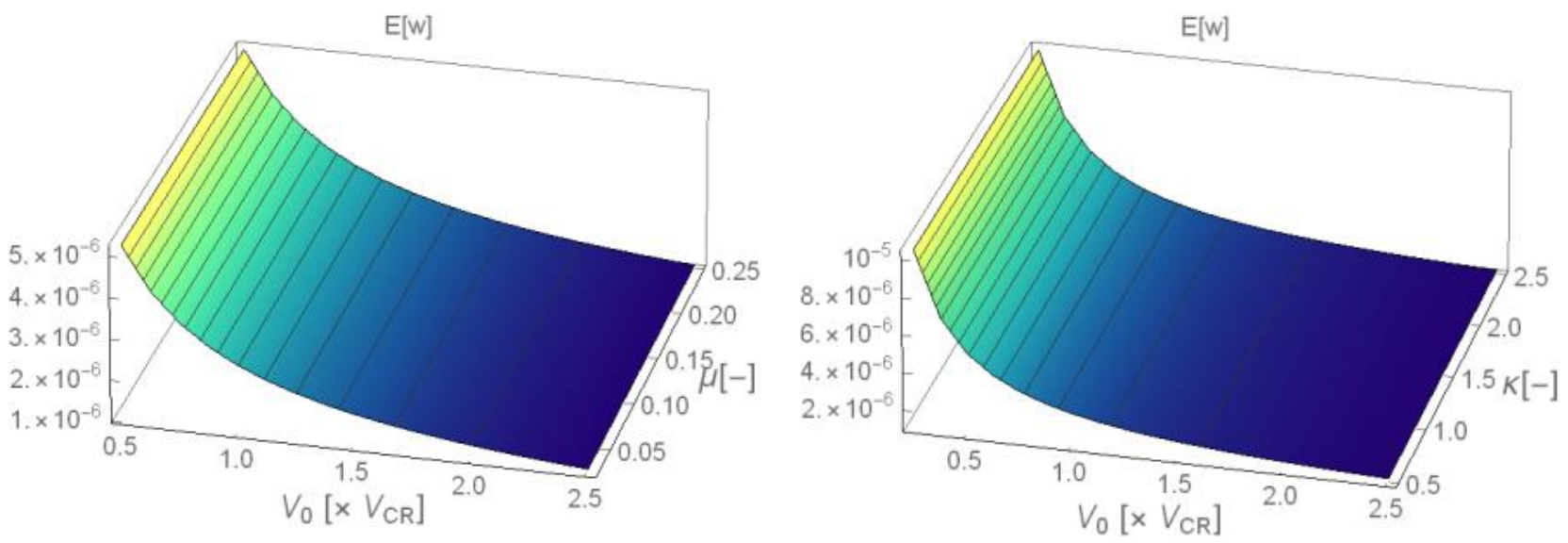

Figure 8: The expected value of deflections in the midspan for different vehicle velocities and also for (a) different parameters $\mu$ of the absorbers and (b) different parameters $K$ of the absorbers.
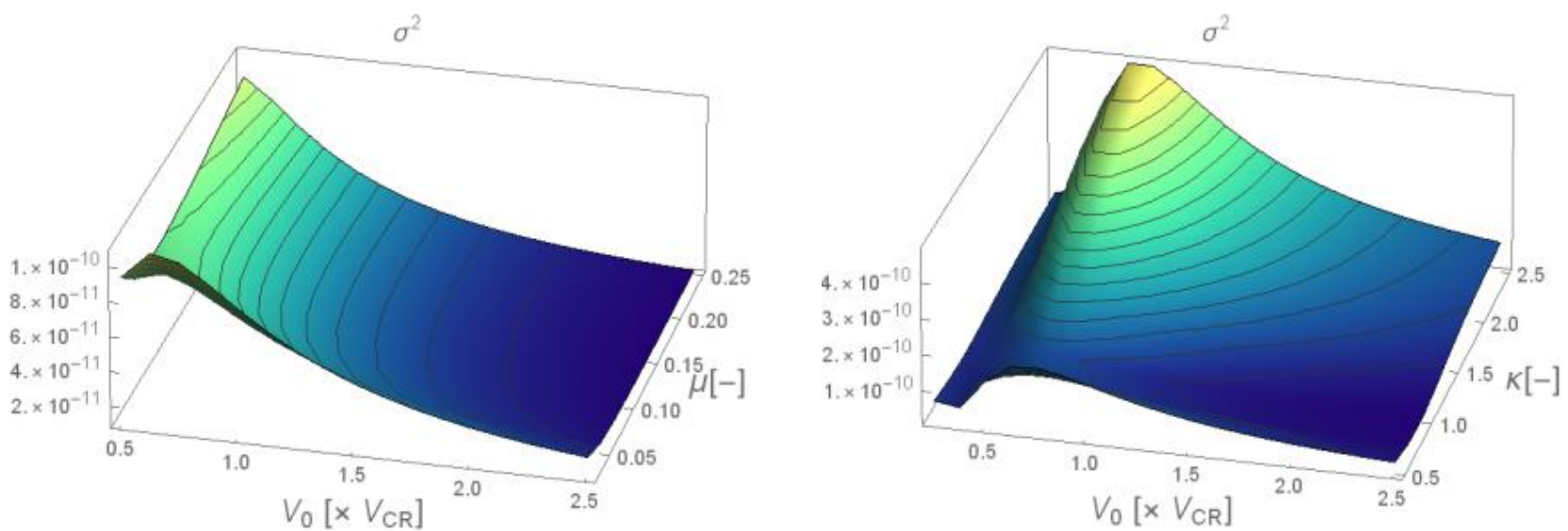

Figure 9: The variance of deflections in the midspan for different vehicle velocities, and also for (a) different parameters $\mu$ of the absorbers and (b) different parameters $\mathrm{K}$ of the absorbers.
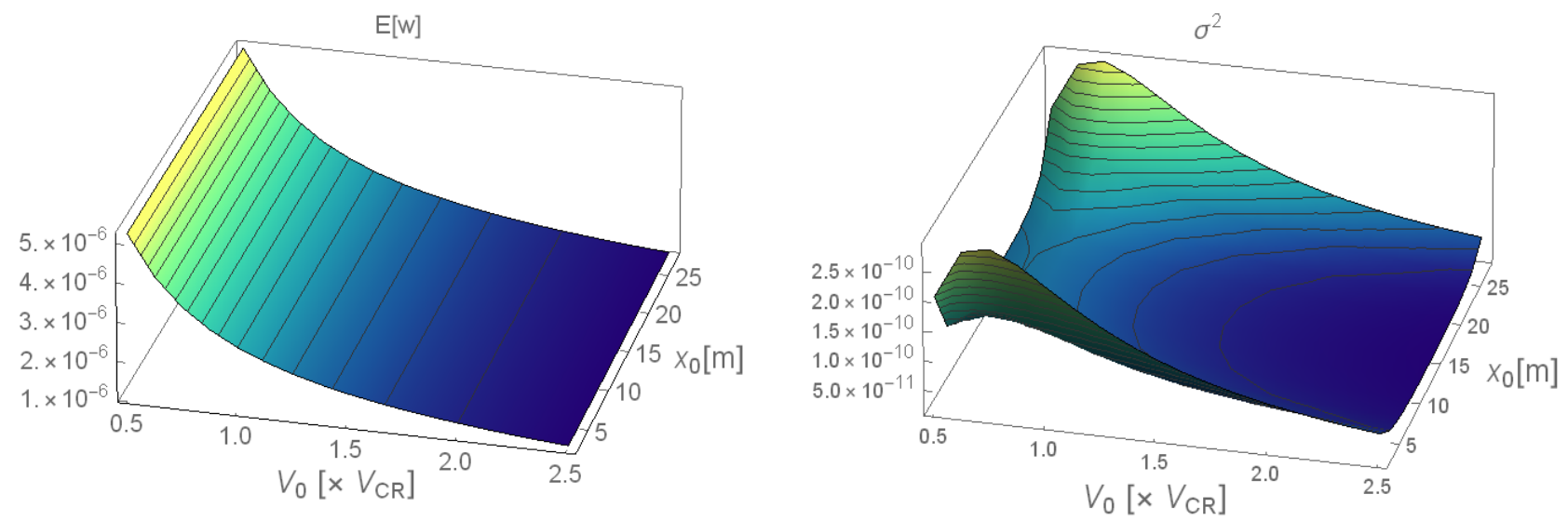

Figure 10: Deflections in the midspan for different locations of the absorber: (a) the expected value and (b) the variance. 
The subject of the research was also the location of the absorber. Is it really the best solution to install the DVA in the middle of the beam? The authors obtained an answer that the optimal operation of the absorber is when it is installed in the range of $0.3-0.6$ of the beam's span. Figure 10 shows the dependence between both the expected value and the variance of the midspan deflection and the absorber's position. The results are presented for different velocities of motor vehicles.

\section{Conclusion and comments}

According to Warburton [40], it is well-known that under the same optimisation procedure, the performance of the TMD system is better with an increase of the total mass of the attached TMD. This study analysed the range of the absorber mass from 0 to as much as $30 \%$ of a structure's mass. Years ago, it was accepted that the total mass of the attached absorbers should not exceed $10 \%$ of the mass of the main system.

After analysing the influence of the absorber's $\mu=M_{a} /$ $m L$ and $\kappa=\omega_{a} / \omega_{s}$ parameters, as well as its location along the length of the bridge beam (together with technical infrastructure devices), it was found that the variance of displacements in the middle of the span should be taken into account when optimising the absorber. The smaller the variance, the better the tuned absorber is. It was found that the expected value of deflection is not significant when tuning the absorber.

In the case of the analysed reinforced concrete beam bridge with a span of $30 \mathrm{~m}$, on which a platform, along with the technical infrastructure, was installed (e.g. hazardous gas), the optimal absorber has parameters in the following ranges: mass $M_{a} \leq 0.05 \mathrm{~mL}, 0.10 \mathrm{~mL}>$ $\left(5 \%-10 \%\right.$ of the total mass of the structure) and $\omega_{a}=\omega_{s}$ (the absorber's frequency is equal to the structure's frequency.

\section{References}

[1] Dallard P., Fitzpatrick T., Flint A., Low A., Smith R.R., Willford M., Roche M., 2001. London Millennium Bridge: PedestrianInduced Lateral Vibration, Journal of Bridge Engineering 6(6), $412-417$.

[2] Yau J.D., Yang Y.B., 2004. Vibration reduction for cable-stayed bridges traveled by high-speed trains, Finite Elements in Analysis and Design 40, 341 - 359.

[3] Majcher K., Wójcicki Z., 2014. Kinematically excited parametric vibration of a tall building model with a TMD. Pt. 1, Numerical analyses. Archives of Civil and Mechanical Engineering 14(1), 204-217.

[4] Herbut A., Rybak J., Brząkała W., 2020. On a Sensor Placement Methodology for Monitoring the Vibrations of Horizontally Excited Ground Sensors 20(7), 1938; https://doi.org/10.3390/ s20071938.

[5] Den Hartog J.P.,1985. Mechanical Vibrations, $4^{\text {th }}$ ed., Dover, New York.

[6] Korenev B. G., Reznikov L.M.,1993. Dynamic vibration absorbers, John Wiley.

[7] Soong T.T., Dargush G.F.,1997. Passive Energy dissipation systems in structural Engineering, Wiley, New York.

[8] Jacquot R. Q., Hoppe D. H.,1973. Optimum random vibration absorbers, Journal of the Engineering Mechanics Division, ASCE 99, 612-616.

[9] Cheung Y.L., Wong W.O.,2013. Optimization of a hybrid vibration absorber for vibration control of structures under force excitation, Journal of Sound and Vibration, 332, 494-509.

[10] Sinha A.,2009, Optimal damped vibration absorber for narrow band random excitations a mixed $\mathrm{H}_{2} / \mathrm{H}_{\infty}$ optimization, Probabilistic Engineering Mechanics 24, 251-254.

[11] Tigli O.F., 2012. Optimum vibration absorber (tuned mass damper) design for linear damped systems subjected to random loads, Journal of Sound and Vibration 331, 3035-3049.

[12] Sieniawska R., Sniady P., Zukowski S., 1996. Optimization of stochastic vibrations absorbers with respect to structure's reliability, Structural Dynamics-EURODYN, Florence, 583-589.

[13] Hua Y., Wong W., Cheng L., 2018. Optimal design of a beambased dynamic vibration absorber using fixed-points theory, Journal of Sound and Vibration 421, 111-131.

[14] Basili M., De Angelis M., Pietrosanti D., 2019. Defective two adjacent single degree of freedom systems linked by springdashpot-inerter for vibration control, Engineering Structures 188, 480-492.

[15] Zuo L., Nayfeh S. A., 2006. The two-degree-of-freedom tunedmass damper for suppression of single-mode vibration under random and harmonic excitation, Journal of Vibration and Acoustics, Transections of the ASME, 128(2), 56-65.

[16] Barredo E., Larios J.G.M., Mayen J., Flores-Hernandez A.A., Colin J., 2019. Optimal design for high-performance passive dynamic vibration absorbers under random vibration, Engineering Structures, 195, 469-489.

[17] Laurentiu M., Agathoklis G., 2014. Optimal design of novel tuned mass-damper-inerter (TMDI) passive vibration control configuration for stochastically support-excited structural systems, Probabilistic Engineering mechanics, 38, 156-164.

[18] Jacquot R.G., 2001. Suppresion of random vibration in plates using vibration absorbers, Journal of Sound and Vibration, 248 (4), 585-596.

[19] Shum K.M., 2015. Tuned vibration absorbers with nonlinear viscous damping for damped structures under random load, Journal of Sound and Vibrations, 346, 70-80.

[20] Javidialesaadi A., Wierschem N.E., 2018. Optimal design of rotational inertial double tuned mass dampers under random excitation, Engineering Structures, 165, 412-421.

[21] Yang F., Sedaghati R., Esmailzadeh E., 2021. Vibration suppression of Structures using tuned mass damper technology: A state-of-the-art review, Journal of Vibration and Control, https://doi.org/10.1177/1077546320984305. 
[22] Frahm H., 1911. Device for damping vibrations of bodies, United States Patent, 3576-3580.

[23] Ormondroyd J., Den Hartog J.P., 1928. The theory of the dynamic vibration absorber, Transactions of ASME, Journal of Applied Mechanics 50 (7), 9-22.

[24] Anh N. D., Nguyen N. X., Hoa L. T., 2013. Design of threeelement dynamic vibration absorber for damped linear structures , Journal of Sound and Vibration 332, 4482-4495.

[25] Asami T., Nishihara O., Baz A.M., 2002. Analytical solutions to $\mathrm{H}_{\infty}$ and $\mathrm{H}_{2}$ optimization of dynamic vibration absorbers attached to damped linear systems, Transactions of ASME Journal of Vibration and Acoustics;124(2), 284-295.

[26] Nishihara 0., Asami T., 2002. Closed-form solutions to the exact optimizations of dynamic vibration absorbers (minimizations of the maximum amplitude magnification factors), Transactions of ASME Journal of Vibration and Acoustics 124(4), 576-582.

[27] Sims N. D., 2007. Vibration absorbers for chatter suppression: a new analytical tuning methodology, Journal of Sound and Vibration 301 (3), 592-607.

[28] Shen Y., Peng H., Li X., Yang S., 2017. Analytically optimal parameters of dynamic vibration absorber with negative stiffness, Mechanical Systems and Signal Processing 85 , 193-203.

[29] Issa J. S., 2013. Vibration absorbers for simply supported beams subjected to constant moving loads. Proceedings of the Institution of Mechanical Engineers Part K Journal of Multi-body Dynamics 226(4):398-404.

[30] Samani F. S., Pellicano F., Masoumi A., 2013. Performances of dynamic vibration absorbers for beams subjected to moving loads. Nonlinear Dynamics 72(1-2).

[31] Crandall S.H. and Mark W.D., 1963. Random Vibration in Mechanical Systems. New York: Academic Press.

[32] Soong T.T., Grigoriu M., 1993. Random vibration of mechanical and structural systems, PTR Prentice-Hall, Inc.

[33] Lin Y.K., Cai G.Q., 1995. Probabilistic structural dynamics: Advanced theory and applications, McGraw-Hill.

[34] Solnes J., 1997. Stochastic processes and random vibrations, John Wiley \& Sons.

[35] Rystwej A., Śniady P., 2007. Dynamic response of an infinite beam and plate to a stochastic train of moving forces, Journal of Sound and Vibration, 299, 1033-1048.

[36] Śniady P., 1989. Dynamic response of linear structures to a random stream of pulses, Journal of Sound and Vibration, 131, 1, 91-102.

[37] Śniady P., 2000. Fundamentals of stochastic structure dynamics (in Polish), Oficyna Wydawnicza Politechniki Wroctawskiej.

[38] Wolfram Mathematica 12. Wolfram Research @Copyright 19882021.

[39] Podwórna M., Grosel J., Śniady P., 2021. Absorbers impact on the reliability of structures subjected to random vibrations, IOP Conference Series: Materials Science and Engineering 1015.

[40] Warburton G.B., 1982. Optimum absorber parameters for various combinations of response and excitation parameters. Earthquake Engineering and Structural Dynamics 10, 381-401. 